\title{
A Study on Finding Optimal Propagation Model Using the Real Radio Spectrum Data in High-Land Area
}

\author{
Ju-Hang Sohn, In-Sung Han*, Minho Park and Duck-Joong Kim ${ }^{1}$ \\ ${ }^{1}$ Agency for Defense and Development for Korea, 31486, Korea \\ spson1@add.re.kr, *p9741181@gmail.com
}

\begin{abstract}
The path loss in wireless communications is influenced by frequency, distance, geographical features, antenna height of transmitting station and the receiving station and artificial environment. The received signal measurement in wireless environment and the path loss prediction model are widely used, because the RF environment is different from region to region and the frequency band is also wide. There is too much cases using $R F$ field measurement to derive the RF model that is costly and time-consuming. Also, theoretical predict models to derive the RF Model and actual frequency environments is often different. Thus, both the field measurement and the theoretical prediction models are complementary to each other. In this paper, we derived the optimal RF model for mountainous region using actual measured data and variety $R F$ model of coverage simulation results. Also we analyzed the correlation of the field measured data with simulation data.
\end{abstract}

Keywords: Path loss, Propagation Model, Coverage Simulation

\section{Introduction}

In accordance with growing needs of wireless communication technology, various research projects related coverage simulation are being carried out for planning optimized service. However, there are some limitations in surveying and analyzing propagation in high-land areas. In order to provide better communication service, various radio environment conditions which are based on wide radio bandwidth (selection of propagation model, correction of value in accordance with radio bandwidth, etc.) should be considered. In an attempt to predict reliable propagation coverage, this paper examines limited propagation data with HTZ Warfare which were collected from high land sites in Korea.

Furthermore, through testing simulations it analyzes comparative performance evaluation for optimized propagation model [1-2].

\section{Radio Measurement Environment}

The measurement was conducted on the basis of the measured high-land Hwacheon site primarily for this study and secondary candidate site (Daebudo, Wonju, Yesan, Hongsung) which performed measurement of radio environment [3], [6-8].

Figure 1 is shown a conFigureuration of transmitting system. Table 1 is shown as a radio measurement information, and the range. The measurement frequency is HF, VHF, UHF band was collected to measure the radio waves from four highlands. Spread form and output was used for the non-modulation (Continuous Wave) CW wave.

* Corresponding Author 
Receiving the operating system was mounted on the vehicle, is the main component composed of a spectrum analyzer, receive antenna, LNA (Low Noise Amp.), GPS (Global Positioning System), a laptop etc.

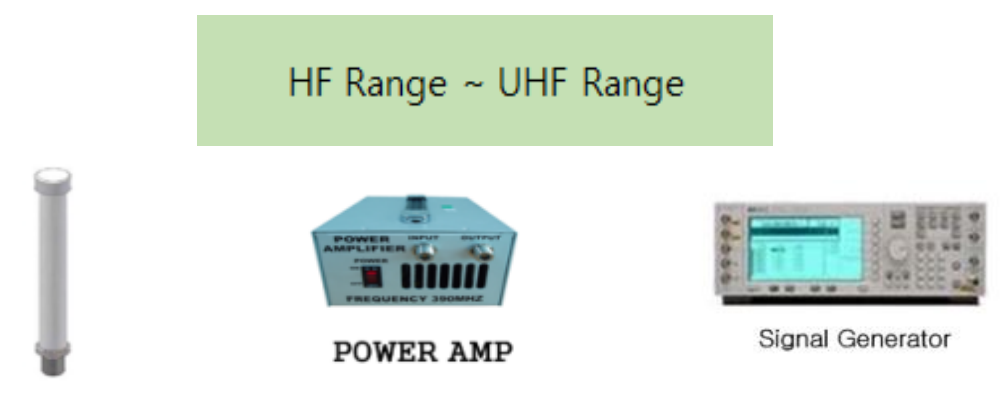

Figure 1. Diagram of Transmitting System

Table 1. Radio Measurement Specification and Coverage

\begin{tabular}{|l|c|}
\hline Category & Contents \\
\hline \hline \multirow{2}{*}{ Frequency } & $\mathrm{HF}(28.7 \mathrm{MHz})$ \\
& $\mathrm{VHF}(110 \mathrm{MHz})$ \\
\hline \multirow{2}{*}{ Location } & $\mathrm{UHF}(415 \mathrm{MHz}, 850 \mathrm{MHz}, 1.8 \mathrm{GHz}, 2.1 \mathrm{GHz})$ \\
\hline Type & -1 st : Whacheon \\
\hline
\end{tabular}

Figure 2 is a structural view for receiving a radio wave signal for measuring the radio wave, and serves to measure and store the received electric field strength according to the moving distance of the vehicle [4-5].

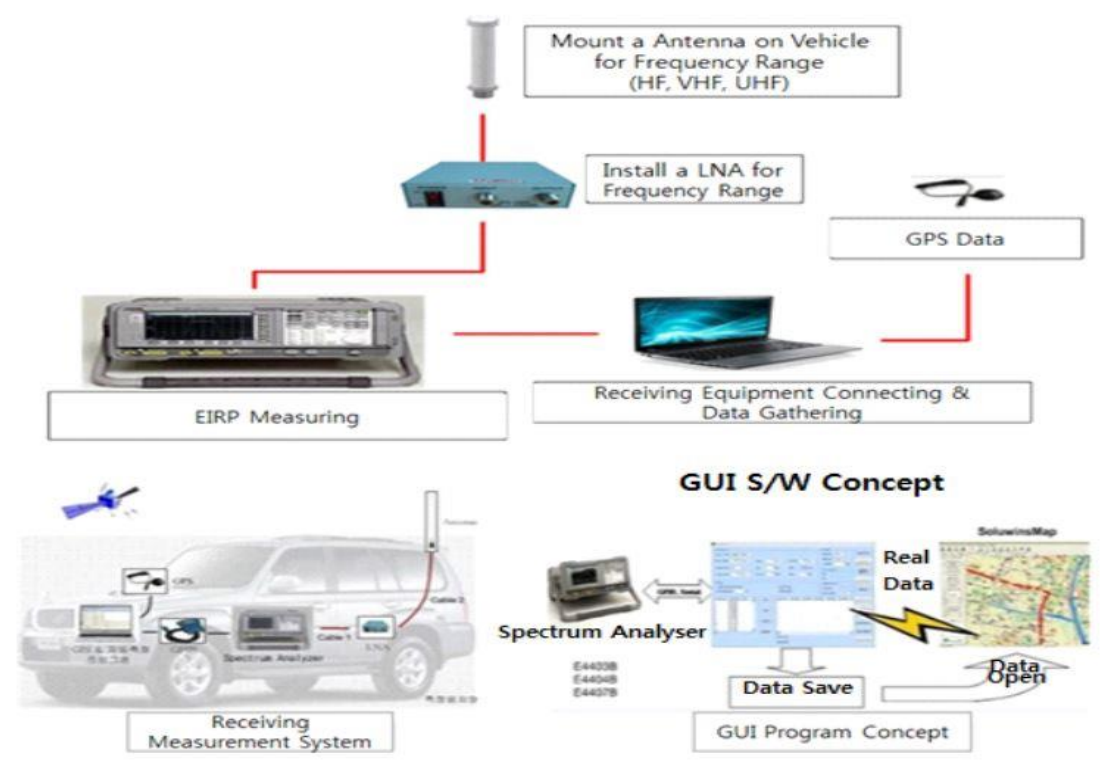

Figure 2. Diagram of Receiving System 


\section{Electric Field Strength Simulation and Correlation Analysis}

Measuring program performs a storing function for the received electric field strength measurement data from the spectrum analyzer and processing the latitude $\&$ longitude data, measurement time through the GPS receiver.
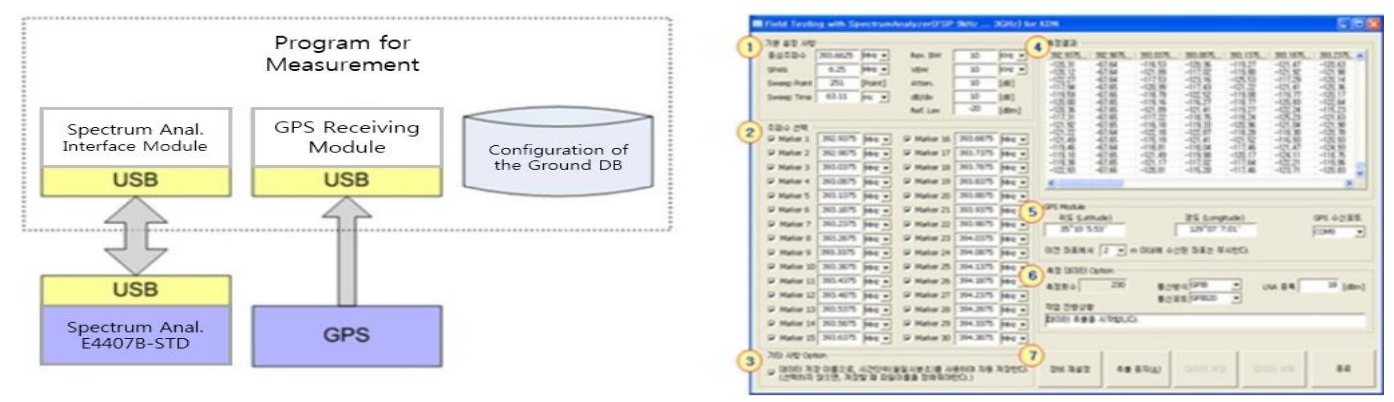

Figure 3. Concept of Measurement Program and Main View Feature

Figure 3 is the information on the GUI screen and the conFigureuration information for a measurement program.

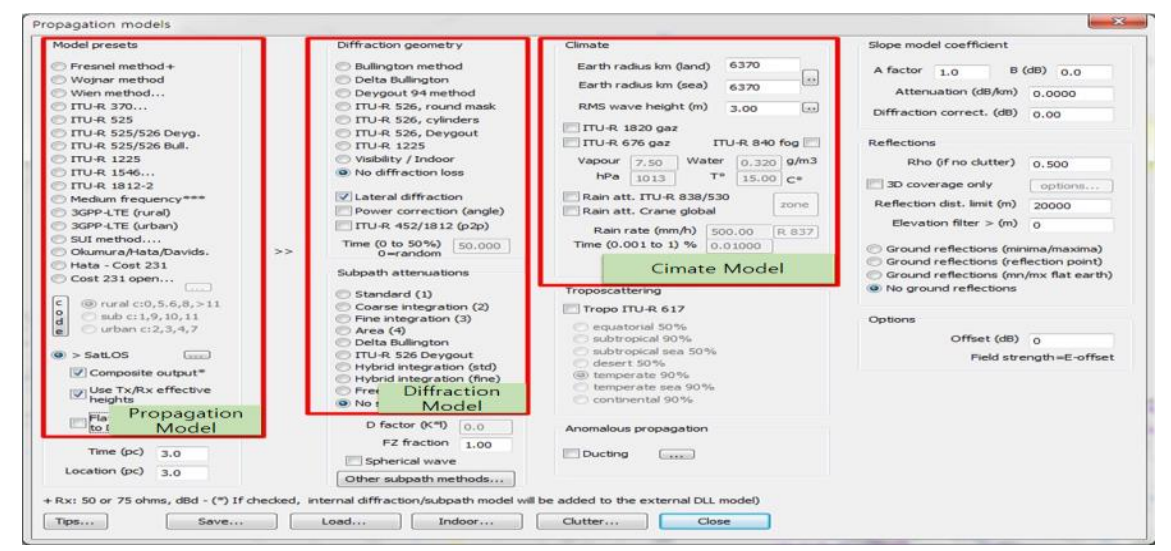

Figure 4. HTZ Warfare Propagation Model Program Feature

Measuring program is transmitted and received and a spectrum analyzer data through the USB interface, and receives the GPS information from the external location information showing that driven by the program.

Figure 4 is information about each of the free space loss model (applied in accordance with the propagation distance attenuation), the diffraction loss model (Non LoS obstructions environment applicable), part path loss attenuation model (Near LoS obstructions environment applicable).

In consideration of the propagation environment of radio waves that target only the broadband measurements and characteristics it may be applied to terrestrial propagation model as described above [9].

\section{Actual Results of Radio Environment}

\subsection{Hwacheon High-Land Area Fixed Radio Measurement Result}

Table 2 shows a fixed radio measurement results are: $8.7 \mathrm{MHz}, 110 \mathrm{MHz}$, $415 \mathrm{MHz}, 850 \mathrm{MHz}, 1.8 \mathrm{GHz}$, and $2.1 \mathrm{GHz}$, respectively, were calculated correlation coefficients of the predicted value and the measured value of the 4 sites. The results 
are respectively $0.26065,0.75378,0.862702,0.118924,0.797126$, and the value of 0.500775 .

The correlation coefficient is related to the contrary case of the perfect match relationship, -1 if the value of $I$ of the range of -1 to $+1,1$, indicates that there is a correlation of 0 .

$28.7 \mathrm{MHz}$ and $2850 \mathrm{MHz}$ portion of the difference between the predicted and measured values were the result of the biggest bands.

This portion is determined by the correction is necessary by all means part. Figure 5 represents the $\mathrm{dB}$ difference between the predicted value and the measured value

Table 2. Radio Measurement Specification and Coverage

\begin{tabular}{|c|c|c|c|c|}
\hline Transmittion & Receive & Distance(m) & Prediciton(dB) & Measured(dB) \\
\hline \hline Hwacheon_28.7 & 1 & 17429.79 & -59.78 & -44.1 \\
\hline Hwacheon_28.7 & 2 & 11538.31 & -73.3 & -60.96 \\
\hline Hwacheon_28.7 & 4 & 15910.13 & -57.29 & -61.26 \\
\hline Hwacheon_28.7 & 8 & 4686.15 & -31.28 & -53.33 \\
\hline Hwacheon_110 & 1 & 17429.79 & -57.83 & -66.4 \\
\hline Hwacheon_110 & 2 & 11538.31 & -72.36 & -73.3 \\
\hline Hwacheon_110 & 4 & 15910.13 & -54.34 & -76.29 \\
\hline Hwacheon_110 & 8 & 4686.15 & -38.7 & -54.77 \\
\hline Hwacheon_415 & 1 & 17429.79 & -68.49 & -58.49 \\
\hline Hwacheon_415 & 2 & 11538.31 & -82.94 & -82.19 \\
\hline Hwacheon_415 & 4 & 15910.13 & -62.8 & -66.15 \\
\hline Hwacheon_415 & 8 & 4686.15 & -48.5 & -55.16 \\
\hline Hwacheon_850 & 1 & 17429.79 & -75.57 & -48.85 \\
\hline Hwacheon_850 & 2 & 11538.31 & -91.74 & -66.16 \\
\hline Hwacheon_850 & 4 & 15910.13 & -68.19 & -77.7 \\
\hline Hwacheon_850 & 8 & 4686.15 & -54.69 & -56.57 \\
\hline Hwacheon_1.8 & 1 & 17429.79 & -83.38 & -78.45 \\
\hline Hwacheon_1.8 & 2 & 11538.31 & -101.08 & -90.66 \\
\hline Hwacheon_1.8 & 4 & 15910.13 & -73.5 & -83.72 \\
\hline Hwacheon_1.8 & 8 & 4686.15 & -61.08 & -77.65 \\
\hline Hwacheon_2.1 & 1 & 17429.79 & -85.05 & -83.69 \\
\hline Hwacheon_2.1 & 2 & 11538.31 & -103.05 & -85.29 \\
\hline Hwacheon_2.1 & 4 & 15910.13 & -74.54 & -89.02 \\
\hline Hwacheon_2.1 & 8 & 4686.15 & -62.41 & -76.33 \\
\hline
\end{tabular}

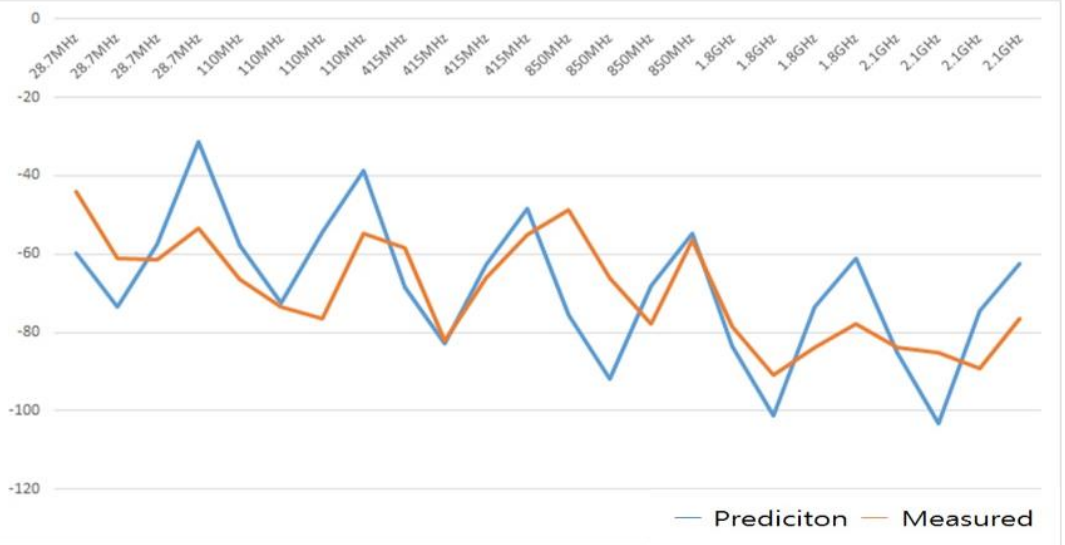

Figure 5. Comparison dB of Prediction and Measurement Value 


\subsection{Frequency-Dependent Correlation Analysis Result}

Table 3. Analysis Result of Each Other Frequencies

\begin{tabular}{|c|c|c|c|c|c|c|}
\hline \multicolumn{2}{|r|}{ Category } & \multirow{2}{*}{$\begin{array}{c}\begin{array}{c}\text { Hwa } \\
\text { cheon }\end{array} \\
\%< \pm 6 \\
\text { dB } \\
\end{array}$} & \multirow{2}{*}{$\begin{array}{c}\begin{array}{c}\text { Daebu } \\
\text { do }\end{array} \\
\%< \pm 6 \\
\text { dB } \\
\end{array}$} & \multirow{2}{*}{$\begin{array}{c}\text { Wonju } \\
\%< \pm 6 \\
\text { dB } \\
\end{array}$} & \multirow{2}{*}{$\begin{array}{c}\text { Yesan } \\
\%< \pm 6 \\
\text { dB } \\
\end{array}$} & \multirow{2}{*}{$\begin{array}{c}\text { Hong } \\
\text { sung } \\
\%< \pm 6 \\
\text { dB } \\
\end{array}$} \\
\hline MHz & Frequency Model & & & & & \\
\hline \multirow{4}{*}{28.7} & $525-526-526$ & 54.51 & 68.44 & 57.04 & 66.73 & 68.27 \\
\hline & 525-deygout94-Standard & 18.66 & 10.33 & 3 & 5.32 & 9.02 \\
\hline & 525-deygout94-NoSubpath & 81.86 & 20.79 & 37.99 & 27.73 & 51.99 \\
\hline & 525-deygout94-coarse & 73 & 69.02 & 57.1 & 75.2 & 60.21 \\
\hline \multirow{4}{*}{110} & $525-526-526$ & - & 85.02 & 38.39 & 82.09 & 58.68 \\
\hline & 525-deygout94-Standard & - & 27.93 & 5.6 & 10.12 & 10.28 \\
\hline & 525-deygout94-NoSubpath & - & 8.79 & 62.41 & 43.1 & 72.49 \\
\hline & 525-deygout94-coarse & - & 81.96 & 46.54 & 86.22 & 37.41 \\
\hline \multirow{4}{*}{380} & $525-526-526$ & 65.28 & 61.43 & 17.74 & 56.33 & 9.99 \\
\hline & 525-deygout94-Standard & 83.8 & 54.85 & 15.19 & 14.06 & 6.81 \\
\hline & 525-deygout94-NoSubpath & 72.36 & 64.16 & 76.61 & 79.77 & 73.67 \\
\hline & 525-deygout94-coarse & 87.41 & 71.08 & 37.76 & 35.69 & 17.54 \\
\hline \multirow{4}{*}{850} & $525-526-526$ & 28.37 & 39.34 & 9.28 & 28.19 & 10.43 \\
\hline & 525-deygout94-Standard & 54.13 & 62.67 & 24.97 & 22.92 & 8.33 \\
\hline & 525-deygout94-NoSubpath & 74.61 & 72.62 & 90.19 & 79.84 & 60.56 \\
\hline & 525-deygout94-coarse & 69.96 & 58.7 & 24.78 & 24.9 & 7.13 \\
\hline \multirow{4}{*}{1800} & $525-526-526$ & 48.04 & 46.26 & 21.34 & 45.82 & 5.2 \\
\hline & 525-deygout94-Standard & 85.61 & 74.73 & 73.93 & 67.99 & 62.31 \\
\hline & 525-deygout94-NoSubpath & 89.63 & 66.82 & 75.11 & 77.24 & 93.03 \\
\hline & 525-deygout94-coarse & 86.96 & 73.32 & 45.19 & 45.82 & 19.53 \\
\hline \multirow{4}{*}{2100} & $525-526-526$ & 44.01 & 49.72 & 22.91 & 57.97 & 5.77 \\
\hline & 525-deygout94-Standard & 83.86 & 72.83 & 72.5 & 76.66 & 65.59 \\
\hline & 525-deygout94-NoSubpath & 89.5 & 65.9 & 69.13 & 68.54 & 92.07 \\
\hline & 525-deygout94-coarse & 84.83 & 76.08 & 45.07 & 57.4 & 23.4 \\
\hline
\end{tabular}



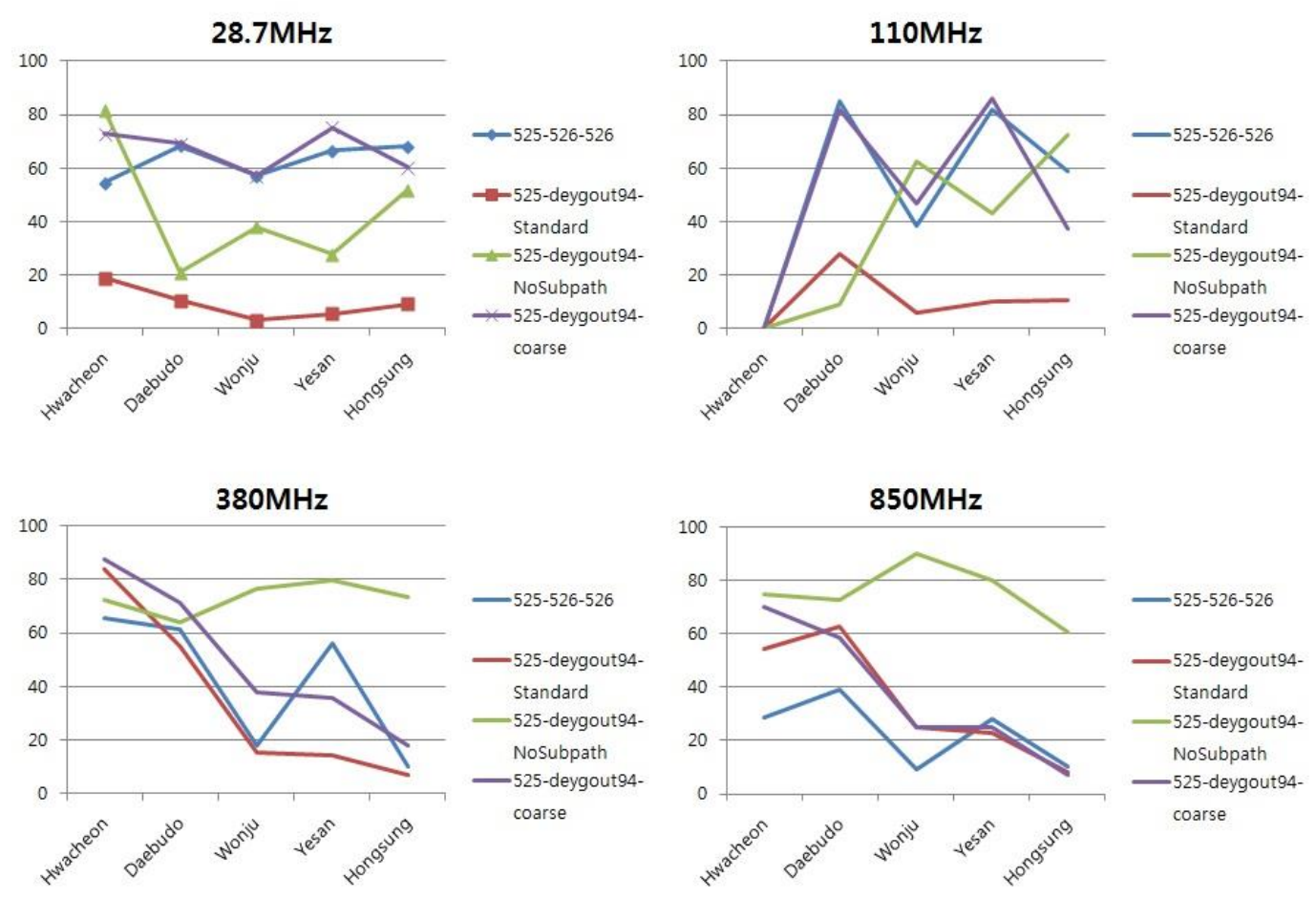

$1800 \mathrm{MHz}$

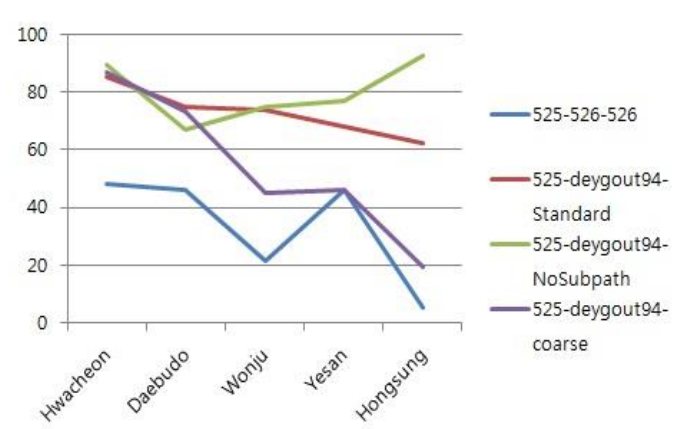

$2100 \mathrm{MHz}$

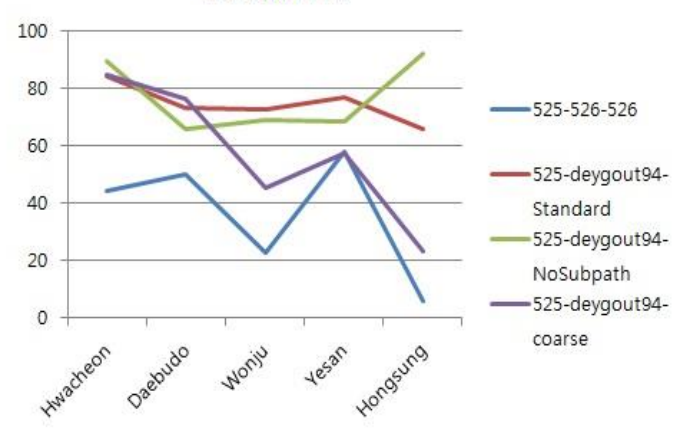

Figure 6. Analysis Result Graph of Each Other Propagation Models

For the frequency base analysis of correlation value, we analyzed radio strength measurement data of each location.

Analysis carried out by propagation model based on the measurement position was recorded to measure targeted at $\%< \pm 6 \mathrm{~dB}$ the data of the comparison result.

As shown in Table 3, 525/526/526 and 525/Deygout94/Coarse model showed a relatively high correlation in $28.7 \mathrm{MHz}$ and $110 \mathrm{MHz}$ bands. $380 \mathrm{MHz}, 850 \mathrm{MHz}$, $1800 \mathrm{MHz}$, and $2100 \mathrm{MHz}$ was generally in 525/Deygout $94 /$ NoSub path model also shows that the correlation appears high. In the case of $1800 \mathrm{MHz}$ and $2100 \mathrm{MHz}$, is effect has been analyzed in 525 / Deygout 94 / Standard Model. Figure 6 shows the data on Table 3 in a graph. 


\subsection{Propagation Model-Dependent Correlation Analysis Result}

Table 4. Analysis Result of Each Other Propagation Models

\begin{tabular}{|c|c|c|c|c|c|c|}
\hline \multicolumn{2}{|c|}{ Category } & Hwacheon & Daebudo & Wonju & Yesan & Hongsung \\
\hline Model & MHz & $\%< \pm 6 \mathrm{~dB}$ & $\%< \pm 6 \mathrm{~dB}$ & $\%< \pm 6 \mathrm{~dB}$ & $\%< \pm 6 \mathrm{~dB}$ & $\%< \pm 6 \mathrm{~dB}$ \\
\hline \multirow{6}{*}{$\begin{array}{c}525-526- \\
526\end{array}$} & 28.7 & 54.51 & 68.44 & 57.04 & 66.73 & 68.27 \\
\hline & 110 & - & 85.02 & 38.39 & 82.09 & 58.68 \\
\hline & 380 & 65.28 & 61.43 & 17.74 & 56.33 & 9.99 \\
\hline & 850 & 28.37 & 39.34 & 9.28 & 28.19 & 10.43 \\
\hline & 1800 & 48.04 & 46.26 & 21.34 & 45.82 & 5.2 \\
\hline & 2100 & 44.01 & 49.72 & 22.91 & 57.97 & 5.77 \\
\hline \multirow{6}{*}{$\begin{array}{c}\text { 525- } \\
\text { deygout } \\
94- \\
\text { Standar } \\
\text { d }\end{array}$} & 28.7 & 18.66 & 10.33 & 3 & 5.32 & 9.02 \\
\hline & 110 & - & 27.93 & 5.6 & 10.12 & 10.28 \\
\hline & 380 & 83.8 & 54.85 & 15.19 & 14.06 & 6.81 \\
\hline & 850 & 54.13 & 62.67 & 24.97 & 22.92 & 8.33 \\
\hline & 1800 & 85.61 & 74.73 & 73.93 & 67.99 & 62.31 \\
\hline & 2100 & 83.86 & 72.83 & 72.5 & 76.66 & 65.59 \\
\hline \multirow{6}{*}{$\begin{array}{c}\text { 525- } \\
\text { deygout } \\
94- \\
\text { NoSubp } \\
\text { ath }\end{array}$} & 28.7 & 81.86 & 20.79 & 37.99 & 27.73 & 51.99 \\
\hline & 110 & - & 8.79 & 62.41 & 43.1 & 72.49 \\
\hline & 380 & 72.36 & 64.16 & 76.61 & 79.77 & 73.67 \\
\hline & 850 & 74.61 & 72.62 & 90.19 & 79.84 & 60.56 \\
\hline & 1800 & 89.63 & 66.82 & 75.11 & 77.24 & 93.03 \\
\hline & 2100 & 89.5 & 65.9 & 69.13 & 68.54 & 92.07 \\
\hline \multirow{6}{*}{$\begin{array}{c}\text { 525- } \\
\text { deygout } \\
\text { 94- } \\
\text { coarse }\end{array}$} & 28.7 & 73 & 69.02 & 57.1 & 75.2 & 60.21 \\
\hline & 110 & - & 81.96 & 46.54 & 86.22 & 37.41 \\
\hline & 380 & 87.41 & 71.08 & 37.76 & 35.69 & 17.54 \\
\hline & 850 & 69.96 & 58.7 & 24.78 & 24.9 & 7.13 \\
\hline & 1800 & 86.96 & 73.32 & 45.19 & 45.82 & 19.53 \\
\hline & 2100 & 84.83 & 76.08 & 45.07 & 57.4 & 23.4 \\
\hline
\end{tabular}

The results relatively low frequency band of Table 4 were 525/526/526, 525 / Deygout94 / Coarse model correlation percentage is high, the high frequency 525 / Deygout94 / Standard., 525 / Deygout94 / NoSubpath correlation model showed that high. Figure 7 shows the data on Table 4 in a graph.

On the other hand, given the wide variety of regional and frequency, is expected to be relatively good for selecting the 525 / Deygout 94 / NoSubpath model. 


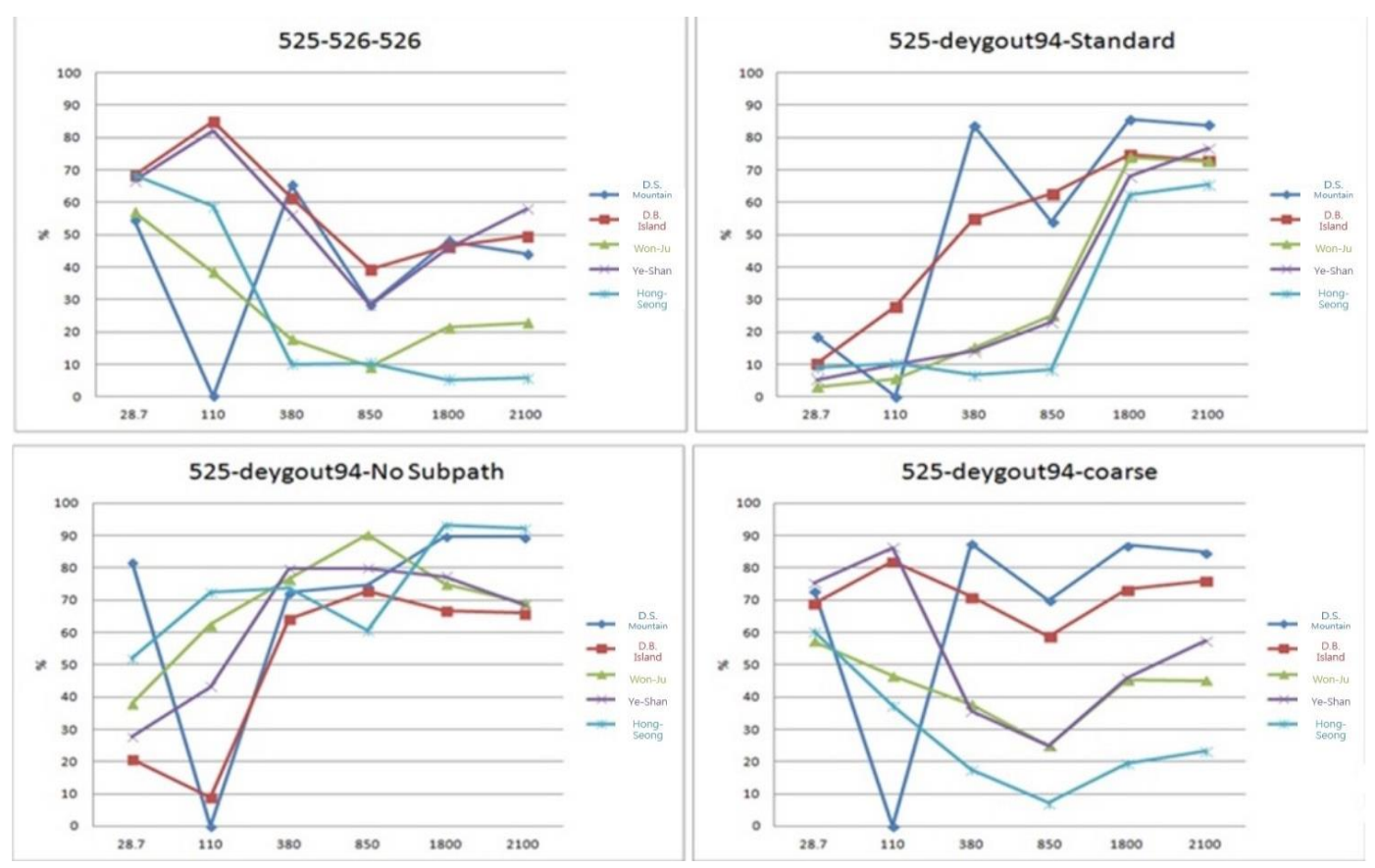

Figure 7. Analysis Result Graph of Each Propagation Models

\section{Conclusion}

Measuring a radio wave environment, the service provider through the survey serves as leading in continuing to provide a good quality of service. Also it can promptly cope with the problems that may occur in detection of new services expected by ensuring the radio environment data on the frequency bands to secure the data required for the allocation of a new service frequency band to the communications network deployment early. This makes it possible to maintain the competitive advantage of network management.

In this paper, we measured the radio spectrum in each High-mountain regions in South Korea. In addition, it can easily and conveniently measure stations during the performance measurement. Also proposed optimized radio wave propagation model through the simulation with HTZ Warfare and analyzed correlation between actual measured data and predicted data.

Based on this study, the sampling data accumulated through continuous spectrum measured will be able to present a reliable optimization model in each region.

\section{References}

[1] J. S. Jeon and Y. W. Kim, "Service analysis and propagation measurement for DGPS land-based reference station in Korea", Journal of the Korean Society of Marine Engineering, vol. 38, no. 4, (2014), pp. 437-443.

[2] B. J. Jang, "Radio Satellite Development trend and plan for 2014", The Journal of The Korean Institute of Communication Sciences, vol. 31, no. 1, (2013), pp. 33-37.

[3] C. Eklund, "IEEE Standard 802.16: A Technical Overview of the WirelessMAN"M Air Interface for Broadband Wireless Access", IEEE Commun. Mag., (2002), pp. 98-107.

[4] H. Park, K. D. Singh and K. Piamrat, "Density-Based Opportunistic Broadcasting Protocol for Emergency Situations in V2X Networks", Journal of Information and Communication Convergence engineering, vol. 12, no. 1, (2014), pp.26-32.

[5] S. Cahyo and J. Jang, "Two-Stage Spectrum Sensing Scheme Using Fussy Logic for Cognitice Radio Networks", Journal of Information and Communication Convergence engineering, vol. 14, no. 1, (2016), pp.1-8.

[6] J. M. Goo, "A study on optimizing prediction model of wave propagation by radio measurement in the small city", Master's thesis of Graduate School of Engineering Hanyang University. 
[7] V. Erceg, "Channel models for fixed wireless access applications," http://www.wirelessman.org/tg3/contrib /802163c-01_29r4.pdf.

[8] Propagation Model Development and Radio Planning for Future WiMAX Systems Deployment in Beirut, America University of Beirut, Final Year Project Spring, (2006).

[9] ATDI, Radio Propagation in ICS telecom, vol. 3, (2009).

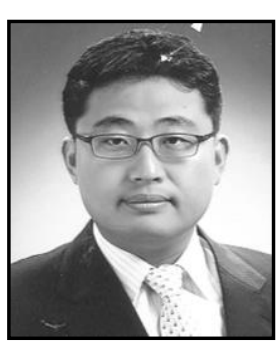

Authors

Ju-Hang Sohn, received his M.S. Degree in Computer Science from Soongsil University, Seoul, Korea, in 2005. From 2005 to 2007 and from 2010 to 2012, he worked as a researcher at ETRI (Electronics and Telecommunications Research Institute). From 2007 to 2010, he worked as a researcher at ADD (Agency for Defense Development). He has been a senior researcher in the Agency for Defense Development (ADD) since 2012. His research interests include network topology, $M \& S$, and wireless telecommunications.

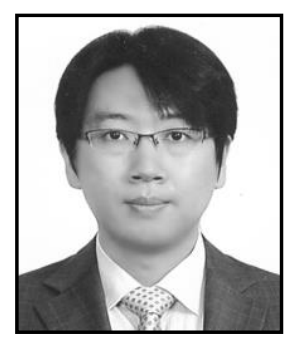

Insung Han, received his M.S. and Ph.D. Degrees in Computer Science from Kwangwoon University, Seoul, Korea, in 2004 and 2009, respectively. From 2010 to 2012, he worked as a senior researcher at R\&D Center of KICA (Korea Information Certificate Authority, Inc). He has been a senior researcher in the Agency for Defense Development (ADD) since 2012. His research interests include network security, ad hoc network routing algorithm, wireless sensor networks security and speech recognition

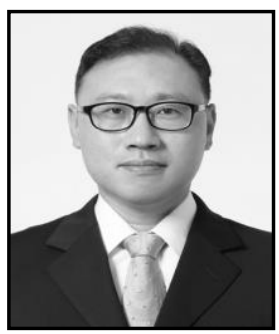

Minho Park, received his B.S. Degree in Information and Communications Engineering from Chungnam National University, Daejeon, Korea, in 2003. The M.S. and Ph. D. degrees are obtained from Gwangju Institute of Science and Technology (GIST), Gwangju, Korea, in the Department of Information and Communications, in 2005 and 2012 respectively. He has been a senior researcher in the Agency for Defense Development (ADD) since 2012. His research interests include heterogeneous communication systems, and neural networks for microwave and optical systems.

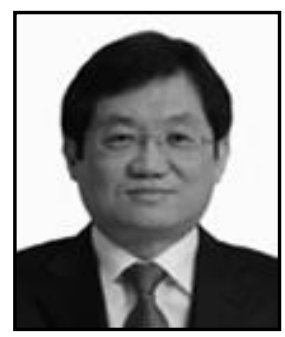

Duckjoong Kim, received his M.S. Degree in Electrical \& Engineering from Yonsei University, Seoul, Korea, in 2006. He has been a principal researcher in the Agency for Defense Development (ADD) since 1988. His research interests include network security, and communication systems. 